\title{
A Numberable Set of Exact Solutions for the Macroscopic Approach to Extended Thermodynamics of Polyatomic Gases with Many Moments
}

\author{
Maria Cristina Carrisi, ${ }^{1}$ Rita Enoh Tchame, ${ }^{2}$ Marcel Obounou, ${ }^{2}$ and Sebastiano Pennisi ${ }^{1}$ \\ ${ }^{1}$ Dipartimento di Matematica ed Informatica, Università di Cagliari, Via Ospedale 72, 09124 Cagliari, Italy \\ ${ }^{2}$ Department of Physics, University of Yaoundé I, Yaoundé, Cameroon \\ Correspondence should be addressed to Sebastiano Pennisi; spennisi@unica.it
}

Received 3 January 2016; Revised 10 April 2016; Accepted 10 July 2016

Academic Editor: Hagen Neidhardt

Copyright (C) 2016 Maria Cristina Carrisi et al. This is an open access article distributed under the Creative Commons Attribution License, which permits unrestricted use, distribution, and reproduction in any medium, provided the original work is properly cited.

\begin{abstract}
A new model for Polyatomic Gases with an arbitrary but fixed number of moments has been recently proposed and investigated in the framework of Extended Thermodynamics; the arbitrariness of the number of moments is linked to a number $N$ and the resulting model is called an $N$-Model. This model has been elaborated in order to take into account the entropy principle, the Galilean relativity principle, and some symmetry conditions. It has been proved that the solution for all these conditions exists, but it has not been written explicitly because hard notation is necessary; it has only been shown how the theory is self-generating in the sense that if we know the closure of the $N$-Model, then we will be able to find that of $(N+1)$-Model. Up to now only a single particular solution has been found in this regard. Instead of this, we find here a numberable set of exact solutions which hold for every fixed number $N$.
\end{abstract}

\section{Introduction}

The model for Polyatomic Gases considers for every number $N$ the following balance equations:

$$
\begin{aligned}
& \partial_{t} F^{i_{1} \cdots i_{n}}+\partial_{k} F^{k i_{1} \cdots i_{n}}=P^{i_{1} \cdots i_{n}} \quad \text { for } n=0, \ldots, N+2 \text {, } \\
& \partial_{t} G^{i_{1} \cdots i_{m}}+\partial_{k} G^{k i_{1} \cdots i_{m}}=Q^{i_{1} \cdots i_{m}} \text { for } m=0, \ldots, N
\end{aligned}
$$

and the resulting model is called an $N$-Model.

Here $F^{i_{1} \cdots i_{n}}$ represents the momentum-like hierarchy, while $G^{i_{1} \cdots i_{m}}$ represents the energy-like hierarchy; the quantities with an index $k$ represent their fluxes, while $P^{i_{1} \cdots i_{n}}$ and $Q^{i_{1} \cdots i_{m}}$ represent the production terms; for the conservation laws of mass and momentum we have $P=0$ and $P^{i}=0$, while for the conservation of energy we have $Q=0$.

This model represents a novelty with respect to the earlier versions of Extended Thermodynamics (ET) which used only $(1)_{1}$ obtaining the important result to have a symmetric hyperbolic system of partial differential equations, with finite speed of propagation of shock waves and other important properties. A very short list of significative articles in this regard is given in [1-7]. These results constituted an important improvement of the models of Ordinary Thermodynamics. A very significative article in this previous framework is given in [8]. But ET was still not completely satisfactory because it is too much restrictive also on the state function relating the pressure $p$ to the mass density $\rho$ and energy density $\epsilon$. This problem has been overcome in Extended Thermodynamics of Polyatomic Gases by considering all the balance equations (1) of which the first one is called the "Mass-Block" of equations and the second is called the "Energy-Block" of equations. See, for example, the articles [9-16].

It has to be noted that in the literature here cited the balance equations are not included in the $\mathrm{N}$-Model, but they can be easily recovered with the theory of subsystems (see [17]) by simply putting the Lagrange multiplier of the last equation in the Mass-Block equal to zero. In [11, 12] it has been shown that the wave speeds of the $N$-Model are the same as those of monatomic gases; but this proof has been 
given with the kinetic equations which are only a subcase of those of the macroscopic approach (in effect, the whole closure here found is zero in the kinetic approach and only parts (5) and (6) remains). In any case there is no problem for what we have said at the beginning of this note. Moreover, a priori the Bhatnagar-Gross-Krook equation (see the equation before (14) in [10]) can be replaced by an infinite hierarchy of balance equations for moments and only for practical reasons some of them are eliminated; consequently, there is no inconsistence if another of these balance equations is eliminated in a second step with the theory of subsystems, as said above. The $N$-Model is here considered because it makes (in the macroscopic approach) the decomposition of tensors in nonconvective parts and in velocity dependent parts more linear. In the kinetic approach this is simply a consequence of the integrals, but in the macroscopic approach here followed this decomposition must be justified in a straightforward manner. In $[11,12]$ the $(M, N)$-system has been proposed with $N \leq M-1$; but the macroscopic approach needs a much heavier notation and this is simplified by the present $N$-Model without losing generality because, in any case, the $(M, N)$-system can be recovered from the present one with the theory of subsystems.

In (1) the independent variables are $F^{i_{1} \cdots i_{n}}$ and $G^{i_{1} \cdots i_{m}}$; the quantities $F^{k i_{1} \cdots i_{n}}$ and $G^{k i_{1} \cdots i_{m}}$ are their corresponding fluxes. The problem will be closed when we know the expressions of $F^{k i_{1} \cdots i_{N+2}}$ and $G^{k i_{1} \cdots i_{N}}$ as functions of the independent variables. Restrictions on their generality are obtained by imposing the entropy principle, the Galilean relativity principle, and the symmetry conditions. These last ones express the fact that each flux in (1) is equal to the independent variable of the subsequent equation, except for the flux in the last equation of the Mass-Block and for that in the last equation of the Energy-Block; for these last fluxes we know only that they are symmetric tensors.

The Entropy Principle, exploited through Liu's Theorem [18] and a bright idea conceived by Ruggeri and Strumia [19], becomes equivalent to assuming the existence of Lagrange Multipliers $\mu_{A}$ and $\lambda_{B}$ which can be taken as independent variables and, after that, we have

$$
\begin{aligned}
F^{i_{1} \cdots i_{n}} & =\frac{\partial h^{\prime}}{\partial \mu_{i_{1} \cdots i_{n}}}, \\
G^{i_{1} \cdots i_{m}} & =\frac{\partial h^{\prime}}{\partial \lambda_{i_{1} \cdots i_{m}}}, \\
F^{k i_{1} \cdots i_{n}} & =\frac{\partial h^{\prime k}}{\partial \mu_{i_{1} \cdots i_{n}}} \\
G^{k i_{1} \cdots i_{m}} & =\frac{\partial h^{\prime k}}{\partial \lambda_{i_{1} \cdots i_{m}}},
\end{aligned}
$$

which expresses all the moments in terms of only two unknown functions, the 4-potential $h^{\prime}$ and $h^{\prime k}$. Their relation with the entropy $h$ and its flux $h^{k}$ is given by

$$
\begin{gathered}
h=-h^{\prime}+F^{i_{1} \cdots i_{n}} \mu_{i_{1} \cdots i_{n}}+G^{i_{1} \cdots i_{m}} \lambda_{i_{1} \cdots i_{m}}, \\
h^{k}=-h^{\prime k}+F^{k i_{1} \cdots i_{n}} \mu_{i_{1} \cdots i_{n}}+G^{k i_{1} \cdots i_{m}} \lambda_{i_{1} \cdots i_{m}} .
\end{gathered}
$$

A nice consequence of (2) is that the field equations assume the symmetric form. Another consequence of (2) is that the above-mentioned symmetry conditions and the Galilean relativity principle can be expressed as

$$
\begin{aligned}
& \frac{\partial h^{\prime k}}{\partial \mu_{i_{1} \cdots i_{n}}}=\frac{\partial h^{\prime}}{\partial \mu_{k i_{1} \cdots i_{n}}} \quad \text { for } n=0, \ldots, N+1 \\
& \frac{\partial h^{\prime[k}}{\partial \mu_{\left.i_{1}\right] i_{2} \cdots i_{N+2}}}=0, \\
& \frac{\partial h^{\prime k}}{\partial \lambda_{i_{1} \cdots i_{m}}}=\frac{\partial h^{\prime}}{\partial \lambda_{k i_{1} \cdots i_{m}}} \quad \text { for } m=0, \ldots, N-1 ; \\
& \frac{\partial h^{\prime[k}}{\partial \lambda_{\left.i_{1}\right] \cdots i_{N}}}=0, \\
& \frac{\partial h^{\prime}}{\partial \mu_{k}} \mu_{i}+\sum_{n=1}^{N+1} \frac{\partial h^{\prime}}{\partial \mu_{k j_{1} \cdots j_{n}}}\left[(n+1) \mu_{i j_{1} \cdots j_{n}}+2 \lambda_{j_{1} \cdots j_{n-1}} \delta_{j_{n} i}\right] \\
& \quad+\sum_{s=0}^{N-1} \frac{\partial h^{\prime}}{\partial \lambda_{k h_{1} \cdots h_{s}}}(s+1) \lambda_{i h_{1} \cdots h_{s}}+h^{\prime} \delta^{k i}=0 .
\end{aligned}
$$

The equivalence of $(4)_{5}$ to the Galilean relativity principle is proved in literature such as $[13,20,21]$ and on Representation Theorems such as that of [22].

In (5), (6), and (7) we report an already known particular solution (see [23]) for the conditions (4). But it is not the general solution; in fact, in the present article we will exhibit a significative set of other solutions depending on a numberable family of arbitrary single variable functions $g_{i}(\lambda)$ and we report it in the next section, (8) and (9). It is expressed through Taylor's expansion around equilibrium which is defined as the state where $\mu_{i_{1} \ldots i_{n}}=0$, for $n=$ $1, \ldots, N+2$, and $\lambda_{i_{1} \cdots i_{m}}=0$, for $m=1, \ldots, N$, so that the only variables which are not zero at equilibrium are $\mu$ and $\lambda$. The first of these is the chemical potential, while $\lambda=1 / 2 T$ with $T$ absolute temperature.

Obviously, also the velocity is nonvanishing at equilibrium; but if we use a decomposition in nonconvective parts and in velocity dependent parts, we obtain the Lagrange multiplier $\mu_{i}$ of the momentum conservation law in terms of the other Lagrange multipliers and we see that its velocity independent part is zero at equilibrium. Moreover, from the fact that the velocity independent parts of $\mu_{\ldots .}$ and $\lambda_{\ldots .}$ are zero at equilibrium, it follows that they are zero also as a whole, without decomposition; the only exceptions are for the Lagrange multipliers of the mass and of the energy conservation laws (see equation (25) of [21], e.g.).

The proof of the fact that (8) and (9) give a solution for (4) is here split into two parts: the first one is reported in Section 3 and the second and final part is described in Section 4.

In conclusion, the sum of the solution reported in (5), (6), and (7) and the present one reported in (8) and (9) is a more general solution for our conditions. 


\section{A Set of Exact Solutions for Conditions (4)}

Before writing our solution we need an apparently complicated notation.

To do the derivatives with respect to $\mu_{i_{1} \cdots i_{n}}$ a number $p_{n}$ of times, we use the compact form $A_{n, 1}$ to indicate a set of $n$ indexes, $A_{n, 2}$ to indicate another set of $n$ indexes, and so on up to $A_{n, p_{n}}$, so that the first index of $A_{\ldots, \ldots}$ indicates how many indexes have the corresponding $\mu_{\ldots}$, while the second index of $A_{\ldots, \ldots}$ indicates how many derivatives we are taking with respect to it. Similarly, to do the derivatives with respect to $\lambda_{i_{1} \cdots i_{m}}$ a number $r_{m}$ of times, we use the compact form $B_{m, 1}$ to indicate a set of $m$ indexes, $B_{m, 2}$ to indicate another set of $m$ indexes, and so on up to $B_{m, r_{m}}$. After describing this notation, we can now report from [23] the already known solution for our conditions and it is

$$
\begin{aligned}
& h^{\prime}=\sum_{\substack{p_{1}, \ldots, p_{N+2}, r_{1}, \ldots, r_{N} \\
p_{1}+2 p_{2}+\cdots+(N+2) p_{N+2} \\
+r_{1}+2 r_{2}+\cdots+N r_{N} \text { even }}}^{0 \cdots \infty} \frac{1}{p_{1} !} \frac{1}{p_{2} !} \cdots \frac{1}{p_{N+2} ! r_{1} !} \frac{1}{r_{2} !} \cdots \frac{1}{r_{N} !} \cdot \frac{\left[1+p_{1}+2 p_{2}+\cdots+(N+2) p_{N+2}+r_{1}+2 r_{2}+\cdots+N r_{N}\right] ! !}{1+p_{1}+2 p_{2}+\cdots+(N+2) p_{N+2}+r_{1}+2 r_{2}+\cdots+N r_{N}} \\
& \cdot \frac{\partial^{1+p_{1}+p_{2}+\cdots+p_{N+2}+r_{1}+r_{2}+\cdots+r_{N}}}{\partial \lambda^{r_{1}+r_{2}+\cdots+r_{N}} \partial \mu^{1+p_{1}+p_{2}+\cdots+p_{N+2}}}\left[\left(\frac{-1}{2 \lambda}\right)^{(1 / 2)\left[p_{1}+2 p_{2}+\cdots+(N+2) p_{N+2}+r_{1}+2 r_{2}+\cdots+N r_{N}\right]} \cdot \psi_{(1 / 2)\left[p_{1}+2 p_{2}+\cdots+(N+2) p_{N+2}+r_{1}+2 r_{2}+\cdots+N r_{N}\right]}\right] \\
& \cdot \delta^{\left(A_{1,1} \cdots A_{1, p_{1}} \cdots A_{N+2,1} \cdots A_{N+2, p_{N+2}} B_{1,1} \cdots B_{1, r_{1}} \cdots B_{N, 1} \cdots B_{N, r_{N}}\right)} \cdot \mu_{A_{1,1}} \cdots \mu_{A_{1, p_{1}}} \cdots \mu_{A_{N+2,1}} \cdots \mu_{A_{N+2, p_{N+2}}} \lambda_{B_{1,1}} \cdots \lambda_{B_{1, r_{1}}} \cdots \lambda_{B_{N, 1}} \cdots \lambda_{B_{N, r_{N}}} \text {, } \\
& h^{\prime k}=\sum_{\substack{p_{1}, \ldots, p_{N+2}, r_{1}, \ldots, r_{N} \\
p_{1}+2 p_{2}+\cdots+(N+2) p_{N+2} \\
+r_{1}+2 r_{2}+\cdots+N r_{N} \text { odd }}}^{0 \cdots \infty} \frac{1}{p_{1} !} \frac{1}{p_{2} !} \cdots \frac{1}{p_{N+2} ! r_{1} ! r_{2} !} \frac{1}{r_{N} !} \cdot\left[p_{1}+2 p_{2}+\cdots+(N+2) p_{N+2}+r_{1}+2 r_{2}+\cdots+N r_{N}\right] ! ! \\
& \cdot \frac{\partial^{1+p_{1}+p_{2}+\cdots+p_{N+2}+r_{1}+r_{2}+\cdots+r_{N}}}{\partial \lambda^{r_{1}+r_{2}+\cdots+r_{N}} \partial \mu^{1+p_{1}+p_{2}+\cdots+p_{N+2}}}\left[\left(\frac{-1}{2 \lambda}\right)^{(1 / 2)\left[1+p_{1}+2 p_{2}+\cdots+(N+2) p_{N+2}+r_{1}+2 r_{2}+\cdots+N r_{N}\right]} \cdot \psi_{(1 / 2)\left[1+p_{1}+2 p_{2}+\cdots+(N+2) p_{N+2}+r_{1}+2 r_{2}+\cdots+N r_{N}\right]}\right] \\
& \cdot \delta^{\left(k A_{1,1} \cdots A_{1, p_{1}} \cdots A_{N+2,1} \cdots A_{N+2, p_{N+2}} B_{1,1} \cdots B_{1, r_{1}} \cdots B_{N, 1} \cdots B_{N, r_{N}}\right)} \cdot \mu_{A_{1,1}} \cdots \mu_{A_{1, p_{1}}} \cdots \mu_{A_{N+2,1}} \cdots \mu_{A_{N+2, p_{N+2}}} \lambda_{B_{1,1}} \cdots \lambda_{B_{1, r_{1}}} \cdots \lambda_{B_{N, 1}} \cdots \lambda_{B_{N, r_{N}}} \text {, }
\end{aligned}
$$

where $\psi_{n}$ is a family of functions depending only on $\mu$ and $\lambda$ and constrained only by

$$
\frac{\partial \psi_{n+1}}{\partial \mu}=\psi_{n}
$$

But the above one is not the unique solution; in fact, by using the same notation, we can now say that a more general solution can be obtained by adding to (5) and (6) the following expressions (8) and (9); that is,

$$
\begin{aligned}
h^{\prime} & =\sum_{\substack{p_{0}, \ldots, p_{N+2}, r_{1}, \ldots, r_{N} \\
\sum_{i=1}^{N+2} i p_{i}+\sum_{i=1}^{N} i r_{i} \text { even }}}^{0 \cdots \infty} \frac{1}{p_{0} !} \frac{1}{p_{1} !} \cdots \frac{1}{p_{N+2} !} \frac{1}{r_{1} !} \frac{1}{r_{2} !} \cdots \frac{1}{r_{N} !} \\
& \cdot \frac{\left[1+\sum_{i=1}^{N+2} i p_{i}+\sum_{i=1}^{N} i r_{i}\right] ! !}{1+\sum_{i=1}^{N+2} i p_{i}+\sum_{i=1}^{N} i r_{i}} \\
& \cdot \frac{\partial^{r_{1}+r_{2}+\cdots+r_{N}}}{\partial \lambda^{r_{1}+r_{2}+\cdots+r_{N}}}\left[\left(\frac{-1}{2 \lambda}\right)^{\sum_{i=0}^{N+2}(3-i) p_{i}-\sum_{i=1}^{N} i r_{i}}\right. \\
& \left.\cdot g_{\sum_{i=0}^{N+2}(i-2) p_{i}+\sum_{i=1}^{N} i r_{i}-1}\right] \\
& \cdot \delta^{\left(A_{1,1} \cdots A_{1, p_{1}} \cdots A_{N+2,1} \cdots A_{N+2, p_{N+2}} B_{1,1} \cdots B_{1, r_{1}} \cdots B_{N, 1} \cdots B_{N, r_{N}}\right)}(\mu)^{p_{0}}
\end{aligned}
$$

$$
\begin{aligned}
& \cdot \mu_{A_{1,1}} \cdots \mu_{A_{1, p_{1}}} \cdots \mu_{A_{N+2,1}} \cdots \mu_{A_{N+2, p_{N+2}}} \lambda_{B_{1,1}} \cdots \lambda_{B_{1, r_{1}}} \\
& \cdots \lambda_{B_{N, 1}} \cdots \lambda_{B_{N, r_{N}}} \\
& h^{\prime k}=\sum_{\substack{p_{0}, \ldots, p_{N+2}, r_{1}, \ldots, r_{N} \\
\sum_{i=1}^{N+2} i p_{i}+\sum_{i=1}^{N} i r_{i} \text { odd }}}^{0 \cdots \infty} \frac{1}{p_{0} !} \frac{1}{p_{1} !} \cdots \frac{1}{p_{N+2} !} \frac{1}{r_{1} !} \\
& \cdot \frac{1}{r_{2} !} \cdots \frac{1}{r_{N} !}\left[\sum_{i=1}^{N+2} i p_{i}+\sum_{i=1}^{N} i r_{i}\right] ! ! \\
& \cdot \frac{\partial^{r_{1}+r_{2}+\cdots+r_{N}}}{\partial \lambda^{r_{1}+r_{2}+\cdots+r_{N}}}\left[\left(\frac{-1}{2 \lambda}\right)^{\sum_{i=0}^{N+2}(3-i) p_{i}-\sum_{i=1}^{N} i r_{i}-1}\right. \\
& \left.\cdot g_{\sum_{i=0}^{N+2}(i-2) p_{i}+\sum_{i=1}^{N} i r_{i}}\right] \\
& \cdot \delta^{\left(k A_{1,1} \cdots A_{1, p_{1}} \cdots A_{N+2,1} \cdots A_{N+2, p_{N+2}} B_{1,1} \cdots B_{1, r_{1}} \cdots B_{N, 1} \cdots B_{N, r_{N}}\right)}(\mu)^{p_{0}} \\
& \cdot \mu_{A_{1,1}} \cdots \mu_{A_{1, p_{1}}} \cdots \mu_{A_{N+2,1}} \cdots \mu_{A_{N+2, p_{N+2}}} \lambda_{B_{1,1}} \cdots \lambda_{B_{1, r_{1}}} \\
& \cdots \lambda_{B_{N, 1}} \cdots \lambda_{B_{N, r_{N}}}
\end{aligned}
$$

where $g_{i}$ is a family of functions depending only on $\lambda$ such that $g_{i}=0$ for $i<0$. Here we use only a letter $\delta^{\cdots}$ as 
a shortened symbol denoting the product of some $\delta^{\cdots}$ each one with 2 indexes and with a final symmetrization over all these indexes.

We note the following:

(i) If $N=0$, the function $g$ in the expression of $h^{\prime k}$ has index $-2 p_{0}-p_{1}$ and consequently this $g$ may be different from zero only if $p_{0}=0$ and $p_{1}=0$; but in this case $p_{1}+2 p_{2}$ is not odd. This fact implies that $h^{\prime k}=0$. Similarly, the function $g$ in the expression of $h^{\prime}$ has index $-2 p_{0}-p_{1}-1$ so that $g=0$ and $h^{\prime}=0$. This confirms the results of [24] for this case $N=0$, because in the sum of (8) and (9) and the corresponding results (5) and (6) of [23] only these last ones remain in the case $N=0$.

(ii) If $N=1$, the function $g$ in the expression of $h^{\prime k}$ has index $-2 p_{0}-p_{1}+p_{3}+r_{1}$ and this is an odd number because $p_{1}+2 p_{2}+3 p_{3}+r_{1}$ must be odd. Consequently, the index $p_{0}$ belongs to the interval $\left[0,\left(-p_{1}+p_{3}+\right.\right.$ $\left.r_{1}-1\right) / 2$ ] if this is not the empty set. By taking into account this fact we see that the sum of the solution reported in (6) and the present expression (9) for the case $N=1$ is the same as that in [24]. Similarly, the function $g$ in the expression of $h^{\prime}$ has index $-2 p_{0}-$ $p_{1}+p_{3}+r_{1}-1$ and this is an odd number because $p_{1}+2 p_{2}+3 p_{3}+r_{1}$ must be even. Consequently, the index $p_{0}$ belongs to the interval $\left[0,\left(-p_{1}+p_{3}+r_{1}-2\right) / 2\right]$ if this is not the empty set. By taking into account this fact we see that the sum of our expression (8) and that in (5) for the case $N=1$ is the same as that in [24].

(iii) If $N \geq 2$ our solution is completely new.

\section{The New Solution (8) and (9) Satisfies (4) $)_{1-4}$}

In this section we prove that (8) and (9) are a solution for $(4)_{1-4}$, putting off $(4)_{5}$ to Section 4 :

(i) Let us substitute firstly (8) and (9) in (4) . We obtain for both sides the following expression:

$$
\begin{aligned}
& \sum_{\substack{p_{0}, \ldots, p_{N+2}, r_{1}, \ldots, r_{N} \\
n+\sum_{i=1}^{N+2} i p_{i}+\sum_{i=1}^{N} i r_{i} \text { odd }}}^{0 \cdots \infty} \frac{1}{p_{0} !} \frac{1}{p_{1} !} \cdots \frac{1}{p_{N+2} !} \frac{1}{r_{1} !} \frac{1}{r_{2} !} \cdots \frac{1}{r_{N} !}[n \\
& \left.+\sum_{i=1}^{N+2} i p_{i}+\sum_{i=1}^{N} i r_{i}\right] ! ! \\
& \cdot \frac{\partial^{r_{1}+r_{2}+\cdots+r_{N}}}{\partial \lambda^{r_{1}+r_{2}+\cdots+r_{N}}}\left[\left(\frac{-1}{2 \lambda}\right)^{\sum_{i=0}^{N+2}(3-i) p_{i}-\sum_{i=1}^{N} i r_{i}-1+3-n}\right. \\
& \left.\cdot g_{\sum_{i=0}^{N+2}(i-2) p_{i}+\sum_{i=1}^{N} i r_{i}+n-2}\right] \\
& \cdot \delta^{\left(k i_{1} \cdots i_{n} A_{1,1} \cdots A_{1, p_{1}} \cdots A_{N+2,1} \cdots A_{N+2, p_{N+2}} B_{1,1} \cdots B_{1, r_{1}} \cdots B_{N, 1} \cdots B_{N, r_{N}}\right)}(\mu)^{p_{0}} \\
& \cdot \mu_{A_{1,1}} \cdots \mu_{A_{1, p_{1}}} \cdots \mu_{A_{N+2,1}} \cdots \mu_{A_{N+2, p_{N+2}}} \lambda_{B_{1,1}} \cdots \lambda_{B_{1, r_{1}}} \\
& \cdots \lambda_{B_{N, 1}} \cdots \lambda_{B_{N, r_{N}}},
\end{aligned}
$$

where we have taken into account that $\mu_{i_{1} \ldots i_{n}}$ here is denoted with $\mu_{A_{n, 1}}$ or with $\mu_{A_{n, 2}}$ and so on up to $\mu_{A_{n, p_{n}}}$. Similarly, $\mu_{k i_{1} \cdots i_{n}}$ is denoted with $\mu_{A_{n+1,1}}$ or with $\mu_{A_{n+1,2}}$ and so on up to $\mu_{A_{n+1, p_{n+1}}}$. Consequently, the left hand side of $(4)_{1}$ causes, with respect to expression (9), a rise of one unity of the index $p_{n}$; similarly, the right hand side of $(4)_{1}$ causes, with respect to expression (8), a rise of one unity of the index $p_{n+1}$.

(ii) It is easy to verify that (9) satisfies $(4)_{2,4}$.

(iii) By substituting (8) and (9) in (4) in the case $m \geq 1$, we obtain for both sides the following expression:

$$
\begin{aligned}
& \sum_{p_{0}, \ldots, p_{N+2}, r_{1}, \ldots, r_{N}}^{0 \cdots \infty} \frac{1}{p_{0} !} \frac{1}{p_{1} !} \cdots \frac{1}{p_{N+2} !} \frac{1}{r_{1} !} \frac{1}{r_{2} !} \cdots \frac{1}{r_{N} !}[m \\
& m+\sum_{i=1}^{N+2} i p_{i}+\sum_{i=1}^{N} i r_{i} \text { odd } \\
& \left.+\sum_{i=1}^{N+2} i p_{i}+\sum_{i=1}^{N} i r_{i}\right] ! ! \\
& \cdot \frac{\partial^{1+r_{1}+r_{2}+\cdots+r_{N}}}{\partial \lambda^{1+r_{1}+r_{2}+\cdots+r_{N}}}\left[\left(\frac{-1}{2 \lambda}\right)^{\sum_{i=0}^{N+2}(3-i) p_{i}-\sum_{i=1}^{N} i r_{i}-1-m}\right. \\
& \left.\cdot g_{\sum_{i=0}^{N+2}(i-2) p_{i}+\sum_{i=1}^{N} i r_{i}+m}\right] \\
& \cdot \delta^{\left(k i_{1} \cdots i_{m} A_{1,1} \cdots A_{1, p_{1}} \cdots A_{N+2,1} \cdots A_{N+2, p_{N+2}} B_{1,1} \cdots B_{1, r_{1}} \cdots B_{N, 1} \cdots B_{N, r_{N}}\right)}(\mu)^{p_{0}} \\
& \cdot \mu_{A_{1,1}} \cdots \mu_{A_{1, p_{1}}} \cdots \mu_{A_{N+2,1}} \cdots \mu_{A_{N+2, p_{N}+2}} \lambda_{B_{1,1}} \cdots \lambda_{B_{1, r_{1}}} \\
& \cdots \lambda_{B_{N, 1}} \cdots \lambda_{B_{N, r_{N}}}
\end{aligned}
$$

where we have taken into account that $\lambda_{i_{1} \cdots i_{m}}$ here is denoted with $\lambda_{B_{m, 1}}$ or with $\lambda_{B_{m, 2}}$ and so on up to $\lambda_{B_{m, r_{m}}}$. Similarly, $\lambda_{k i_{1} \cdots i_{m}}$ is denoted with $\lambda_{B_{m+1,1}}$ or with $\lambda_{B_{m+1,2}}$ and so on up to $\lambda_{B_{m+1, r_{m+1}}}$. Consequently, the left hand side of $(4)_{3}$ with $m \geq 1$ causes, with respect to expression (9), a rise of one unity of the index $r_{m}$; similarly, the right hand side of $(4)_{3}$ with $m \geq 1$ causes, with respect to expression (8), a rise of one unity of the index $r_{m+1}$.

(iv) Let us conclude substituting (8) and (9) into $(4)_{3}$ in the case $m=0$.

We have the same situation of the above case for its right hand side, while for its left hand side we have to take simply the derivative of (9) with respect to $\lambda$. More precisely, we obtain for both sides the following expression:

$$
\begin{gathered}
\sum_{\substack{p_{0}, \ldots, p_{N+2}, r_{1}, \ldots, r_{N} \\
\sum_{i=1}^{N+2} i p_{i}+\sum_{i=1}^{N} i r_{i} \text { odd }}}^{0 \cdots \infty} \frac{1}{p_{0} !} \frac{1}{p_{1} !} \cdots \frac{1}{p_{N+2} !} \frac{1}{r_{1} !} \\
\cdot \frac{1}{r_{2} !} \cdots \frac{1}{r_{N} !}\left[\sum_{i=1}^{N+2} i p_{i}+\sum_{i=1}^{N} i r_{i}\right] ! !
\end{gathered}
$$




$$
\begin{aligned}
& \cdot \frac{\partial^{1+r_{1}+r_{2}+\cdots+r_{N}}}{\partial \lambda^{1+r_{1}+r_{2}+\cdots+r_{N}}}\left[\left(\frac{-1}{2 \lambda}\right)^{\sum_{i=0}^{N+2}(3-i) p_{i}-\sum_{i=1}^{N} i r_{i}-1}\right. \\
& \left.\cdot g_{\sum_{i=0}^{N+2}(i-2) p_{i}+\sum_{i=1}^{N} i r_{i}}\right] \\
& \cdot \delta^{\left(k A_{1,1} \cdots A_{1, p_{1}} \cdots A_{N+2,1} \cdots A_{N+2, p_{N+2}} B_{1,1} \cdots B_{1, r_{1}} \cdots B_{N, 1} \cdots B_{N, r_{N}}\right)}(\mu)^{p_{0}} \\
& \cdot \mu_{A_{1,1}} \cdots \mu_{A_{1, p_{1}}} \cdots \mu_{A_{N+2,1}} \cdots \mu_{A_{N+2, p_{N+2}}} \lambda_{B_{1,1}} \cdots \lambda_{B_{1, r_{1}}} \\
& \cdots \lambda_{B_{N, 1}} \cdots \lambda_{B_{N, r_{N}}} \cdot
\end{aligned}
$$

This concludes our proof that $(4)_{1-4}$ are satisfied by (8) and (9). In the next section we will consider $(4)_{5}$.

\section{The New Solution (8) and (9) Satisfies (4)}

First of all we note that $(4)_{5}$ can be rewritten as

$$
\begin{aligned}
& \sum_{n=0}^{N+1} \frac{\partial h^{\prime}}{\partial \mu_{k j_{1} \cdots j_{n}}}(n+1) \mu_{i j_{1} \cdots j_{n}}+\frac{\partial h^{\prime}}{\partial \mu_{k i}} 2 \lambda \\
& \quad+\sum_{s=0}^{N-1} 2 \lambda_{j_{1} \cdots j_{s+1}} \frac{\partial h^{\prime}}{\partial \mu_{k j_{1} \cdots j_{s+1} i}} \\
& \quad+\sum_{s=0}^{N-1} \frac{\partial h^{\prime}}{\partial \lambda_{k h_{1} \cdots h_{s}}}(s+1) \lambda_{i h_{1} \cdots h_{s}}+h^{\prime} \delta^{k i}=0 .
\end{aligned}
$$

(i) After that, we note that the first term of this relation thanks to (8) becomes

$$
\begin{aligned}
& \sum_{n=0}^{N+1}(n+1) \mu_{i j_{1} \cdots j_{n}} \frac{\partial h^{\prime}}{\partial \mu_{k j_{1} \cdots j_{n}}}=\sum_{n=0}^{N+1}(n+1) p_{n+1} \cdot \sum_{\substack{p_{0}, \ldots, p_{N+2}, r_{1}, \ldots, r_{N} \\
\sum_{i=1}^{N+2} i p_{i}+\sum_{i=1}^{N} i r_{i} \text { even }}}^{0 \cdots \infty} \frac{1}{p_{0} !} \frac{1}{p_{1} !} \cdots \frac{1}{p_{N+2} !} \frac{1}{r_{1} !} \frac{1}{r_{2} !} \cdots \frac{1}{r_{N} !} \\
& \cdot \frac{\left[1+\sum_{i=1}^{N+2} i p_{i}+\sum_{i=1}^{N} i r_{i}\right] ! !}{1+\sum_{i=1}^{N+2} i p_{i}+\sum_{i=1}^{N} i r_{i}} \cdot \frac{\partial^{r_{1}+r_{2}+\cdots+r_{N}}}{\partial \lambda^{r_{1}+r_{2}+\cdots+r_{N}}}\left[\left(\frac{-1}{2 \lambda}\right)^{\sum_{i=0}^{N+2}(3-i) p_{i}-\sum_{i=1}^{N} i r_{i}} g_{\sum_{i=0}^{N+2}(i-2) p_{i}+\sum_{i=1}^{N} i r_{i}-1}\right] \\
& \cdot(\mu)^{p_{0}} \cdot \delta^{j_{n+1} i} \delta^{\left(k j_{1} \cdots j_{n} A_{1,1} \cdots A_{1, p_{1}} \cdots A_{n+1,1} \cdots A_{n+1, p_{n+1}-1} \cdots A_{N+2,1} \cdots A_{N+2, p_{N+2}} B_{1,1} \cdots B_{1, r_{1}} \cdots B_{N, 1} \cdots B_{N, r_{N}}\right)} \cdot \mu_{A_{1,1}} \\
& \cdots \mu_{A_{1, p_{1}}} \cdots \mu_{A_{n+1,1}} \cdots \mu_{A_{n+1, p_{n+1}-1}} \mu_{j_{1} \cdots j_{n+1}} \cdots \mu_{A_{N+2,1}} \cdots \mu_{A_{N+2, p_{N+2}}} \cdot \lambda_{B_{1,1}} \cdots \lambda_{B_{1, r_{1}}} \cdots \lambda_{B_{N, 1}} \cdots \lambda_{B_{N, r_{N}}} \text {, }
\end{aligned}
$$

because the derivation causes a presence of the factor $p_{n+1}$ and the substitution of $\mu_{A_{n+1,1}} \cdots \mu_{A_{n+1, p_{n+1}}}$ with $\mu_{A_{n+1,1}} \cdots \mu_{A_{n+1, p_{n+1}-1}}$ and the new free indexes $k j_{1} \cdots j_{n}$ in the expression of $\delta$; moreover, we have also substituted $\mu_{i j_{1} \cdots j_{n}}$ with $\mu_{j_{1} \cdots j_{n+1}} \delta^{j_{n+1} i}$. Now in the resulting expression we can insert a symmetrization over all the indexes of the set $j_{1} \cdots j_{n+1} A_{n+1,1} \cdots A_{n+1, p_{n+1}-1}$ because that expression remains the same if we exchange two of these indexes. This fact is evident if the two indexes are taken between $j_{1} \cdots j_{n+1}$; for the proof in the other cases, let us consider the shortened expression $\delta^{j_{n+1} i} \delta^{\left(k j_{1} \cdots j_{n} k_{1} \cdots k_{n} k_{n+1} \cdots\right)} \mu_{j_{1} \cdots j_{n+1}} \mu_{k_{1} \cdots k_{n+1}}$; here we can exchange the names of the indexes $j$. with those of $k_{\text {. }}$, so that the above shortened expression becomes $\delta^{k_{n+1} i} \delta^{\left(k k_{1} \cdots k_{n} j_{1} \cdots j_{n} j_{n+1} \cdots\right)} \mu_{k_{1} \cdots k_{n+1}} \mu_{j_{1} \cdots j_{n+1}}$.

Now we can exchange the indexes $k_{1} \cdots k_{n}$ with the indexes $j_{1} \cdots j_{n}$ in the expression of $\delta^{\left(k k_{1} \cdots k_{n} j_{1} \cdots j_{n} j_{n+1} \cdots\right)}$ because this is a symmetric tensor.

We obtain $\delta^{k_{n+1} i} \delta^{\left(k j_{1} \cdots j_{n} k_{1} \cdots k_{n} j_{n+1} \cdots\right)} \mu_{k_{1} \cdots k_{n+1}} \mu_{j_{1} \cdots j_{n+1}}$. By comparing this result with the expression which we started from, it is the same if we had exchanged the indexes $j_{n+1}$ and $k_{n+1}$. This completes the proof of the fact that expression (14) remains the same if we exchange two indexes of the set $j_{1} \cdots j_{n+1} A_{n+1,1} \cdots A_{n+1, p_{n+1}-1}$; so we can insert there a symmetrization over those indexes and (14) becomes

$$
\begin{aligned}
\sum_{n=0}^{N+1}(n+1) \mu_{i j_{1} \cdots j_{n}} \frac{\partial h^{\prime}}{\partial \mu_{k j_{1} \cdots j_{n}}}= & \sum_{n=0}^{N+1}(n+1) p_{n+1} \cdot \sum_{\begin{array}{c}
p_{0}, \cdots, p_{N+2}, r_{1}, \ldots, r_{N} \\
\sum_{i=1}^{N+2} i p_{i}+\sum_{i=1}^{N} i r_{i} \text { even }
\end{array}}^{0 \cdots \infty} \frac{1}{p_{0} !} \frac{1}{p_{1} !} \cdots \frac{1}{p_{N+2} !} \frac{1}{r_{1} !} \frac{1}{r_{2} !} \cdots \frac{1}{r_{N} !} \\
& \frac{\left[1+\sum_{i=1}^{N+2} i p_{i}+\sum_{i=1}^{N} i r_{i}\right] ! !}{1+\sum_{i=1}^{N+2} i p_{i}+\sum_{i=1}^{N} i r_{i}} \cdot \frac{\partial^{r_{1}+r_{2}+\cdots+r_{N}}}{\partial \lambda^{r_{1}+r_{2}+\cdots+r_{N}}}\left[\left(\frac{-1}{2 \lambda}\right)^{\sum_{i=0}^{N+2}(3-i) p_{i}-\sum_{i=1}^{N} i r_{i}} g_{\sum_{i=0}^{N+2}(i-2) p_{i}+\sum_{i=1}^{N} i r_{i}-1}\right] \\
& \cdot(\mu)^{p_{0}} \cdot \delta \frac{j_{n+1} i}{\delta} \delta^{\left(k j_{1} \cdots j_{n} A_{1,1} \cdots A_{1, p_{1}} \cdots A_{n+1,1} \cdots A_{n+1, p_{n+1}-1} \cdots A_{N+2,1} \cdots A_{N+2, p_{N+2}} B_{1,1} \cdots B_{1, r_{1}} \cdots B_{N, 1} \cdots B_{N, r_{N}}\right)} \cdot \mu_{A_{1,1}} \\
\cdots & \mu_{A_{1, p_{1}}} \cdots \mu_{A_{n+1,1}} \cdots \mu_{A_{n+1, p_{n+1}-1}} \mu_{j_{1} \cdots j_{n+1}} \cdots \mu_{A_{N+2,1}} \cdots \mu_{A_{N+2, p_{N+2}}} \cdot \lambda_{B_{1,1}} \cdots \lambda_{B_{1, r_{1}}} \cdots \lambda_{B_{N, 1}} \cdots \lambda_{B_{N, r_{N}}},
\end{aligned}
$$


where underlined indexes denote symmetrization over these indexes. Now we observe that $(n+1) p_{n+1}$ is exactly the number of the indexes of the set $j_{1} \cdots j_{n+1} A_{n+1,1}$ $\cdots A_{n+1, p_{n+1}-1}$ and that, thanks to the summation $\sum_{n=0}^{N+1}$, the index near $i$ in $\delta^{j_{n+1} i}$ can be every index of the set $A_{1,1} \cdots A_{1, p_{1}} \cdots A_{n+1,1} \cdots A_{n+1, p_{n+1}} \cdots A_{N+2,1} \cdots A_{N+2, p_{N+2}}$. These facts allow rewriting (15) as

$$
\begin{aligned}
\sum_{n=0}^{N+1}(n+1) \mu_{i j_{1} \cdots j_{n}} \frac{\partial h^{\prime}}{\partial \mu_{k j_{1} \cdots j_{n}}}= & {\left[\sum_{n=0}^{N+1}(n+1) p_{n+1}\right] \cdot \sum_{\substack{p_{0}, \cdots, p_{N+2}, r_{1}, \cdots, r_{N} \\
\sum_{i=1}^{N+2} i p_{i}+\sum_{i=1}^{N} i r_{i} \text { even }}}^{0 \cdots \cdots \infty} \frac{1}{p_{0} !} \frac{1}{p_{1} !} \cdots \frac{1}{p_{N+2} !} \frac{1}{r_{1} !} \frac{1}{r_{2} !} \cdots \frac{1}{r_{N} !} } \\
& \cdot \frac{\left[1+\sum_{i=1}^{N+2} i p_{i}+\sum_{i=1}^{N} i r_{i}\right] ! !}{1+\sum_{i=1}^{N+2} i p_{i}+\sum_{i=1}^{N} i r_{i}} \cdot \frac{\partial^{r_{1}+r_{2}+\cdots+r_{N}}}{\partial \lambda^{r_{1}+r_{2}+\cdots+r_{N}}}\left[\left(\frac{-1}{2 \lambda}\right)^{\sum_{i=0}^{N+2}(3-i) p_{i}-\sum_{i=1}^{N} i r_{i}} g_{\sum_{i=0}^{N+2}(i-2) p_{i}+\sum_{i=1}^{N} i r_{i}-1}\right] \\
& \cdot(\mu)^{p_{0}} \cdot \delta^{\frac{j_{n+1}}{i}} \delta^{\left(k j_{1} \cdots j_{n} A_{1,1} \cdots A_{1, p_{1}} \cdots A_{n+1,1} \cdots A_{n+1, p_{n+1}-1} \cdots A_{N+2,1} \cdots A_{N+2, p_{N+2}} B_{1,1} \cdots B_{1, r_{1}} \cdots B_{N, 1} \cdots B_{N, r_{N}}\right)} \cdot \mu_{A_{1,1}} \\
& \cdots \mu_{A_{1, p_{1}}} \cdots \mu_{A_{n+1,1}} \cdots \mu_{A_{n+1, p_{n+1}-1}} \mu_{j_{1} \cdots j_{n+1}} \cdots \mu_{A_{N+2,1}} \cdots \mu_{A_{N+2, p_{N+2}}} \cdot \lambda_{B_{1,1}} \cdots \lambda_{B_{1, r_{1}}} \cdots \lambda_{B_{N, 1}} \cdots \lambda_{B_{N, r_{N}}} .
\end{aligned}
$$

(ii) For the fourth term of (13) we can do similar passages (the difference is that we have $\lambda_{\ldots}$ instead of $\mu_{\ldots}, N-2$ instead of $N$, and $s$ instead of $n$ ); in this way that term becomes

$$
\begin{aligned}
& \sum_{s=0}^{N-1}(s+1) \lambda_{i h_{1} \cdots h_{s}} \frac{\partial h^{\prime}}{\partial \lambda_{k h_{1} \cdots h_{s}}}=\left[\sum_{s=0}^{N-1}(s+1) r_{s+1}\right] \cdot \sum_{\substack{p_{0}, \ldots, p_{N+2,}, r_{1}, \ldots, r_{N} \\
\sum_{i=1}^{N+2} i p_{i}+\sum_{i=1}^{N} r_{i} \text { even }}}^{0, \cdots \infty} \frac{1}{p_{0} !} \frac{1}{p_{1} !} \cdots \frac{1}{p_{N+2} !} \frac{1}{r_{1} !} \frac{1}{r_{2} !} \cdots \frac{1}{r_{N} !} \\
& \cdot \frac{\left[1+\sum_{i=1}^{N+2} i p_{i}+\sum_{i=1}^{N} i r_{i}\right] ! !}{1+\sum_{i=1}^{N+2} i p_{i}+\sum_{i=1}^{N} i r_{i}} \cdot \frac{\partial^{r_{1}+r_{2}+\cdots+r_{N}}}{\partial \lambda^{r_{1}+r_{2}+\cdots+r_{N}}}\left[\left(\frac{-1}{2 \lambda}\right)^{\sum_{i=0}^{N+2}(3-i) p_{i}-\sum_{i=1}^{N} i r_{i}} g_{\sum_{i=0}^{N+2}(i-2) p_{i}+\sum_{i=1}^{N} i r_{i}-1}\right]
\end{aligned}
$$

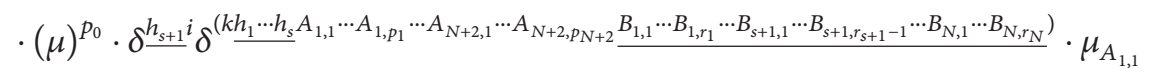

$$
\begin{aligned}
& \cdots \mu_{A_{1, p_{1}}} \cdots \mu_{A_{N+2,1}} \cdots \mu_{A_{N+2, p_{N+2}}} \cdot \lambda_{B_{1,1}} \cdots \lambda_{B_{1, r_{1}}} \cdots \lambda_{B_{s+1,1}} \cdots \lambda_{B_{s+1, r_{s+1}-1}} \cdots \lambda_{B_{N, 1}} \cdots \lambda_{B_{N, r_{N}}} .
\end{aligned}
$$

(iii) If we look at the last term of (13), we see that it can be written together with (16) and (17) and they become

$$
\begin{aligned}
& \sum_{n=0}^{N+1} \frac{\partial h^{\prime}}{\partial \mu_{k j_{1} \cdots j_{n}}}(n+1) \mu_{i j_{1} \cdots j_{n}}+\sum_{s=0}^{N-1} \frac{\partial h^{\prime}}{\partial \lambda_{k h_{1} \cdots h_{s}}}(s+1) \\
& \cdot \lambda_{i h_{1} \cdots h_{s}}+h^{\prime} \delta^{k i}=\left[1+\sum_{n=0}^{N+1}(n+1) p_{n+1}\right. \\
& \left.+\sum_{s=0}^{N-1}(s+1) r_{s+1}\right] \cdot \sum_{\substack{p_{0} \ldots, p_{N+2,2}, \ldots, r_{N} \\
\sum_{i=1}^{N+2} i p_{i}+\sum_{i=1}^{N} i r_{i} \text { even }}}^{0 \cdots \infty} \frac{1}{p_{0} !} \frac{1}{p_{1} !} \\
& \quad \ldots \frac{1}{p_{N+2} !} \frac{1}{r_{1} !} \frac{1}{r_{2} !} \cdots \frac{1}{r_{N} !} \cdot \frac{\left[1+\sum_{i=1}^{N+2} i p_{i}+\sum_{i=1}^{N} i r_{i}\right] ! !}{1+\sum_{i=1}^{N+2} i p_{i}+\sum_{i=1}^{N} i r_{i}} \\
& \cdot \frac{\partial^{r_{1}+r_{2}+\cdots+r_{N}}}{\partial \lambda^{r_{1}+r_{2}+\cdots+r_{N}}}\left[\left(\frac{-1}{2 \lambda}\right)^{\sum_{i=0}^{N+2}(3-i) p_{i}-\sum_{i=1}^{N} i r_{i}}\right.
\end{aligned}
$$

$$
\begin{aligned}
& \left.\cdot g_{\sum_{i=0}^{N+2}(i-2) p_{i}+\sum_{i=1}^{N} i r_{i}-1}\right](\mu)^{p_{0}} \\
& \left.\cdot \delta^{k i} \delta \frac{\left(A_{1,1} \cdots A_{1, p_{1}} \cdots A_{N+2,1} \cdots A_{N+2, p_{N+2}} B_{1,1} \cdots B_{1, r_{1}} \cdots B_{N, 1} \cdots B_{N, r_{N}}\right.}{}\right) \\
& \cdot \mu_{A_{1,1}} \cdots \mu_{A_{1, p_{1}}} \cdots \mu_{A_{N+2,1}} \cdots \mu_{A_{N+2, p_{N+2}}} \lambda_{B_{1,1}} \cdots \lambda_{B_{1, r_{1}}} \\
& \cdots \lambda_{B_{N, 1}} \cdots \lambda_{B_{N, r_{N}}},
\end{aligned}
$$

where also the index $k$ has been put under the symmetrization and we recall that a property of symmetrization is $\delta^{i \underline{k}} \delta^{(\cdots)}=$ $\delta^{i \underline{k}} \delta^{\prime-}=\delta^{(i k \cdots)}$. We note that the coefficient in square bracket at the beginning of the right hand side of (18) has become equal to the denominator of the half factorial of that right hand side.

(iv) For the second term of (13), we can use (8) and the derivation causes someway the rising of one unity of the index $p_{2}$; the result is 


$$
\begin{aligned}
& \frac{\partial h^{\prime}}{\partial \mu_{k i}} 2 \lambda=2 \lambda \sum_{\substack{p_{0}, \ldots, p_{N+2}, r_{1}, \ldots, r_{N} \\
\sum_{i=1}^{N+2} i p_{i}+\sum_{i=1}^{N} i r_{i} \text { even }}}^{0 \cdots \infty} \frac{1}{p_{0} !} \frac{1}{p_{1} !} \cdots \frac{1}{p_{N+2} !} \frac{1}{r_{1} !} \frac{1}{r_{2} !} \\
& \cdots \frac{1}{r_{N} !} \cdot\left[1+\sum_{i=1}^{N+2} i p_{i}+\sum_{i=1}^{N} i r_{i}\right] ! ! \\
& \cdot \frac{\partial^{r_{1}+r_{2}+\cdots+r_{N}}}{\partial \lambda^{r_{1}+r_{2}+\cdots+r_{N}}}\left[\left(\frac{-1}{2 \lambda}\right)^{\sum_{i=0}^{N+2}(3-i) p_{i}-\sum_{i=1}^{N} i r_{i}+1}\right. \\
& \left.\cdot g_{\sum_{i=0}^{N+2}(i-2) p_{i}+\sum_{i=1}^{N} i r_{i}-1}\right](\mu)^{p_{0}} \\
& \cdot \delta^{\left(k i A_{1,1} \cdots A_{1, p_{1}} \cdots A_{N+2,1} \cdots A_{N+2, p_{N+2}} B_{1,1} \cdots B_{1, r_{1}} \cdots B_{N, 1} \cdots B_{N, r_{N}}\right)} \\
& \cdot \mu_{A_{1,1}} \cdots \mu_{A_{1, p_{1}}} \cdots \mu_{A_{N+2,1}} \cdots \mu_{A_{N+2, p_{N+2}}} \lambda_{B_{1,1}} \cdots \lambda_{B_{1, r_{1}}} \\
& \cdots \lambda_{B_{N, 1}} \cdots \lambda_{B_{N, r_{N}}} \cdot
\end{aligned}
$$

(v) There remains considering the third term of (13); for it we can use (8) and the derivation causes someway the rising of one unity of the index $p_{s+3}$; in this way it becomes

$$
\begin{aligned}
& \sum_{s=0}^{N-1} 2 \lambda_{j_{1} \cdots j_{s+1}} \frac{\partial h^{\prime}}{\partial \mu_{k j_{1} \cdots j_{s+1} i}}=\sum_{s=0}^{N-1} 2 \lambda_{j_{1} \cdots j_{s+1}} \\
& \sum_{p_{0}, \ldots, p_{N+2}, r_{1}, \ldots, r_{N}}^{0 \cdots \infty} \frac{1}{p_{0} !} \frac{1}{p_{1} !} \cdots \frac{1}{p_{N+2} !} \frac{1}{r_{1} !} \frac{1}{r_{2} !} \\
& s+3+\sum_{i=1}^{N+2} i p_{i}+\sum_{i=1}^{N} i r_{i} \text { even } \\
& \cdots \frac{1}{r_{N} !} \cdot \frac{\left[s+4+\sum_{i=1}^{N+2} i p_{i}+\sum_{i=1}^{N} i r_{i}\right] ! !}{s+4+\sum_{i=1}^{N+2} i p_{i}+\sum_{i=1}^{N} i r_{i}} \\
& \cdot \frac{\partial^{r_{1}+r_{2}+\cdots+r_{N}}}{\partial \lambda^{r_{1}+r_{2}+\cdots+r_{N}}}\left[\left(\frac{-1}{2 \lambda}\right)^{-s+\sum_{i=0}^{N+2}(3-i) p_{i}-\sum_{i=1}^{N} i r_{i}}\right. \\
& \left.\cdot g_{s+1+\sum_{i=0}^{N+2}(i-2) p_{i}+\sum_{i=1}^{N} i r_{i}-1}\right](\mu)^{p_{0}} \\
& \cdot \delta^{\left(k j_{1} \cdots j_{s+1} i A_{1,1} \cdots A_{1, p_{1}} \cdots A_{N+2,1} \cdots A_{N+2, p_{N+2}} B_{1,1} \cdots B_{1, r_{1}} \cdots B_{N, 1} \cdots B_{N, r_{N}}\right)} \\
& \cdot \mu_{A_{1,1}} \cdots \mu_{A_{1, p_{1}}} \cdots \mu_{A_{N+2,1}} \cdots \mu_{A_{N+2, p_{N+2}}} \lambda_{B_{1,1}} \cdots \lambda_{B_{1, r_{1}}} \\
& \cdots \lambda_{B_{N, 1}} \cdots \lambda_{B_{N, r_{N}}} \text {. }
\end{aligned}
$$

In this expression, we can substitute $\lambda_{j_{1} \cdots j_{s+1}}$ with $\lambda_{B_{s+1, r_{s+1}+1}}$ and $j_{1} \cdots j_{s+1}$ with $B_{s+1, r_{s+1}+1}$; moreover, we substitute $1 /\left(r_{s+1}\right.$ !) with $\left(r_{s+1}+1\right) /\left(r_{s+1}+1\right)$ ! and, after that, decrease $r_{s+1}$ of one unity (which is equivalent to a change of index). So this expression becomes

$$
\begin{aligned}
& \sum_{s=0}^{N-1} 2 \lambda_{j_{1} \cdots j_{s+1}} \frac{\partial h^{\prime}}{\partial \mu_{k j_{1} \cdots j_{s+1} i}}=\sum_{s=0}^{N-1} 2 r_{s+1} \\
& \cdot \sum_{\substack{p_{0}, \ldots, p_{N+2}, r_{1}, \ldots, r_{N} \\
2+\sum_{i=1}^{N+2} i p_{i}+\sum_{i=1}^{N} i r_{i} \text { even }}}^{0 \cdots \infty} \frac{1}{p_{0} !} \frac{1}{p_{1} !} \cdots \frac{1}{p_{N+2} ! r_{1} !} \frac{1}{r_{2} !} \cdots \frac{1}{r_{N} !}
\end{aligned}
$$

$$
\begin{aligned}
& \cdot \frac{\left[3+\sum_{i=1}^{N+2} i p_{i}+\sum_{i=1}^{N} i r_{i}\right] ! !}{3+\sum_{i=1}^{N+2} i p_{i}+\sum_{i=1}^{N} i r_{i}} \\
& \cdot \frac{\partial^{-1+r_{1}+r_{2}+\cdots+r_{N}}}{\partial \lambda^{-1+r_{1}+r_{2}+\cdots+r_{N}}}\left[\left(\frac{1}{2 \lambda}\right)^{1+\sum_{i=0}^{N+2}(3-i) p_{i}-\sum_{i=1}^{N} i r_{i}}\right. \\
& \left.\cdot g_{\sum_{i=0}^{N+2}(i-2) p_{i}+\sum_{i=1}^{N} i r_{i}-1}\right](\mu)^{p_{0}} \\
& \cdot \delta^{\left(k i A_{1,1} \cdots A_{1, p_{1}} \cdots A_{N+2,1} \cdots A_{N+2, p_{N+2}} B_{1,1} \cdots B_{1, r_{1}} \cdots B_{N, 1} \cdots B_{N, r_{N}}\right)} \\
& \cdot \mu_{A_{1,1}} \cdots \mu_{A_{1, p_{1}}} \cdots \mu_{A_{N+2,1}} \cdots \mu_{A_{N+2, p_{N+2}}} \lambda_{B_{1,1}} \cdots \lambda_{B_{1, r_{1}}} \\
& \cdots \lambda_{B_{N, 1}} \cdots \lambda_{B_{N, r_{N}}} \cdot
\end{aligned}
$$

It is true that with change of index we have in the summation the extra term with $r_{s+1}=0$; but it does not effect the result for the presence of the coefficient $r_{s+1}$.

If we look at the expressions (18), (19), and (21) we conclude that to prove (13) it is sufficient that the following relation holds:

$$
\begin{aligned}
& \frac{\partial^{r_{1}+r_{2}+\cdots+r_{N}}}{\partial \lambda^{r_{1}+r_{2}+\cdots+r_{N}}}\left[\left(\frac{-1}{2 \lambda}\right)^{\sum_{i=0}^{N+2}(3-i) p_{i}-\sum_{i=1}^{N} i r_{i}}\right. \\
& \left.\cdot g_{\sum_{i=0}^{N+2}(i-2) p_{i}+\sum_{i=1}^{N} i r_{i}-1}\right] \\
& +2 \lambda \frac{\partial^{r_{1}+r_{2}+\cdots+r_{N}}}{\partial \lambda^{r_{1}+r_{2}+\cdots+r_{N}}}\left[\left(\frac{-1}{2 \lambda}\right)^{1+\sum_{i=0}^{N+2}(3-i) p_{i}-\sum_{i=1}^{N} i r_{i}}\right. \\
& \left.\cdot g_{\sum_{i=0}^{N+2}(i-2) p_{i}+\sum_{i=1}^{N} i r_{i}-1}\right]+\sum_{s=0}^{N-1} 2 r_{s+1} \frac{\partial^{-1+r_{1}+r_{2}+\cdots+r_{N}}}{\partial \lambda^{-1+r_{1}+r_{2}+\cdots+r_{N}}} \\
& \quad\left[\left(\frac{-1}{2 \lambda}\right)^{1+\sum_{i=0}^{N+2}(3-i) p_{i}-\sum_{i=1}^{N} i r_{i}} g_{\sum_{i=0}^{N+2}(i-2) p_{i}+\sum_{i=1}^{N} i r_{i}-1}\right] \\
& \quad=0,
\end{aligned}
$$

and to prove this relation it is sufficient to prove that

$$
\frac{\partial^{r}}{\partial \lambda^{r}} \vartheta+2 \lambda \frac{\partial^{r}}{\partial \lambda^{r}}\left(\frac{-1}{2 \lambda} \vartheta\right)+2 r \frac{\partial^{-1+r}}{\partial \lambda^{-1+r}}\left(\frac{-1}{2 \lambda} \vartheta\right)=0,
$$

where we have put $r=r_{1}+r_{2}+\cdots+r_{N}$ and $\vartheta=$ $(-1 / 2 \lambda)^{\sum_{i=0}^{N+2}(3-i) p_{i}-\sum_{i=1}^{N} i r_{i}} g_{\sum_{i=0}^{N+2}(i-2) p_{i}+\sum_{i=1}^{N} i r_{i}-1}$.

Obviously, (23) is an identity because we have

$$
\begin{aligned}
\frac{\partial^{r}}{\partial \lambda^{r}} \vartheta & =\frac{\partial^{r}}{\partial \lambda^{r}}\left(-2 \lambda \frac{-1}{2 \lambda} \vartheta\right) \\
& =-2 \lambda \frac{\partial^{r}}{\partial \lambda^{r}}\left(\frac{-1}{2 \lambda} \vartheta\right)-2 r \frac{\partial^{-1+r}}{\partial \lambda^{-1+r}}\left(\frac{-1}{2 \lambda} \vartheta\right) .
\end{aligned}
$$

This concludes all the arguments which we had to prove. 


\section{Conclusions}

We conclude that, in the particular case $N=0$, we obtain the 11 moments' model and the solution here indicated is exactly that already obtained in literature with the macroscopic approach. In the case $N=1$ we have that the present one is a particular solution for that already known in literature. So we have seen how it transfers itself also to higher values of $N$. In this way we are approaching the goal to obtain the most general solution and this will be the object of a future research.

\section{Competing Interests}

The authors declare that they have no competing interests.

\section{References}

[1] I.-S. Liu and I. Müller, "Extended thermodynamics of classical and degenerate ideal gases," Archive for Rational Mechanics and Analysis, vol. 83, no. 4, pp. 285-332, 1983.

[2] I.-S. Liu, I. Müller, and T. Ruggeri, "Relativistic thermodynamics of gases," Annals of Physics, vol. 169, no. 1, pp. 191-219, 1986.

[3] I. Müller and T. Ruggeri, Rational Extended Thermodynamics, Springer Tracts in Natural Philosophy, Springer, New York, NY, USA, 2nd edition, 1998.

[4] I. Müller, "Entropy and energy,-a universal competition," Entropy, vol. 10, no. 4, pp. 462-476, 2008.

[5] M. C. Carrisi, "A macroscopic solution for a model suggested by the non relativistic limit of relativistic extended thermodynamics," International Journal of Pure and Applied Mathematics, vol. 67, no. 3, pp. 291-325, 2011.

[6] M. C. Carrisi and S. Pennisi, "Extended thermodynamics of charged gases with many moments: an alternative closure," Journal of Mathematical Physics, vol. 54, no. 9, Article ID 093101, 15 pages, 2013.

[7] M. C. Carrisi, A. Farci, M. Obounou, and S. Pennisi, "Relativistic extended thermodynamics from the Lagrangian view-point," Ricerche di Matematica, vol. 64, no. 2, pp. 357-376, 2015.

[8] A. Atangana and B. S. T. Alkahtani, "Analysis of the Keller-Segel model with a fractional derivative without singular kernel," Entropy, vol. 17, no. 6, pp. 4439-4453, 2015.

[9] T. Arima, S. Taniguchi, T. Ruggeri, and M. Sugiyama, "Extended thermodynamics of dense gases," Continuum Mechanics and Thermodynamics, vol. 24, no. 4-6, pp. 271-292, 2012.

[10] T. Arima, S. Taniguchi, T. Ruggeri, and M. Sugiyama, "Extended thermodynamics of real gases with dynamic pressure: an extension of Meixner's theory," Physics Letters A, vol. 376, no. 44, pp. 2799-2803, 2012.

[11] T. Arima, A. Mentrelli, and T. Ruggeri, "Molecular extended thermodynamics of rarefied polyatomic gases and wave velocities for increasing number of moments," Annals of Physics, vol. 345, pp. 111-140, 2014.

[12] T. Ruggeri and M. Sugiyama, Rational extended thermodynamics beyond the monatomic gas, Springer, Berline, Germany, 2015.

[13] M. C. Carrisi and S. Pennisi, "An 18 moments model for dense gases: entropy and Galilean relativity principles without expansions," Entropy, vol. 17, no. 1, pp. 214-230, 2015.

[14] M. C. Carrisi and S. Pennisi, "Extended thermodynamics for dense gases up to whatever order," International Journal of NonLinear Mechanics, vol. 77, pp. 74-84, 2015.
[15] M. C. Carrisi, R. Enoh Tchame, M. Obounou, and S. Pennisi, "Extended thermodynamics for dense gases up to whatever order and with only some symmetries," Entropy, vol. 17, no. 10, pp. 7052-7075, 2015.

[16] M. C. Carrisi, S. Pennisi, T. Ruggeri, and M. Sugiyama, "Extended thermodynamics of dense gases in the presence of dynamic pressure," Ricerche di Matematica, vol. 64, no. 2, pp. 403-419, 2015.

[17] G. Boillat and T. Ruggeri, "Hyperbolic principal subsystems: entropy convexity and subcharacteristic conditions," Archive for Rational Mechanics and Analysis, vol. 137, no. 4, pp. 305-320, 1997.

[18] I.-S. Liu, "Method of Lagrange multipliers for exploitation of the entropy principle," Archive for Rational Mechanics and Analysis, vol. 46, pp. 131-148, 1972.

[19] T. Ruggeri and A. Strumia, "Main field and convex covariant density for quasi-linear hyperbolic systems. Relativistic fluid dynamics," Annales de l'Institut Henri Poincaré, vol. 34, no. 1, pp. 65-84, 1981.

[20] T. Ruggeri, "Galilean invariance and entropy principle for systems of balance laws. The structure of extended thermodynamics," Continuum Mechanics and Thermodynamics, vol. 1, no. 1, pp. 3-20, 1989.

[21] S. Pennisi and T. Ruggeri, "A new method to exploit the entropy principle and Galilean invariance in the macroscopic approach of extended thermodynamics," Ricerche di Matematica, vol. 55, no. 2, pp. 319-339, 2006.

[22] M. C. Carrisi, S. Montisci, and S. Pennisi, "Representation theorems in a 4-dimensional Euclidean space. The case with only skew-symmetric tensors," International Journal of Pure and Applied Mathematics, vol. 93, no. 6, pp. 929-979, 2014.

[23] M. C. Carrisi, R. Enoh Tchame, M. Obounou, and S. Pennisi, "An exact solution for the macroscopic approach to extended thermodynamics of dense gases with many moments," International Journal of Pure and Applied Mathematics, vol. 106, no. 1, pp. 171-189, 2016.

[24] M. C. Carrisi, S. Pennisi, and J. M. Sellier, "Extended thermodynamics of dense gases with at least 24 moments," Ricerche di Matematica, 2016. 


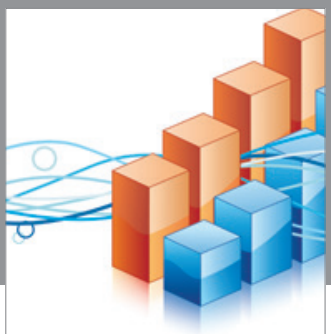

Advances in

Operations Research

vatem alat4

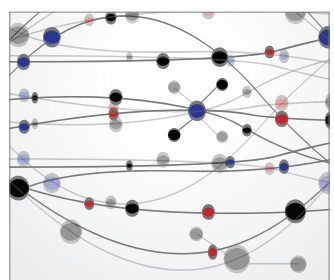

\section{The Scientific} World Journal
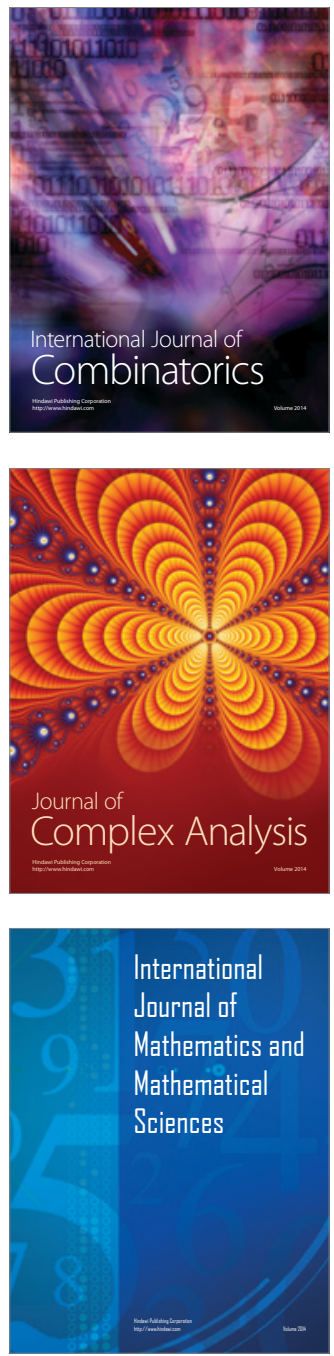
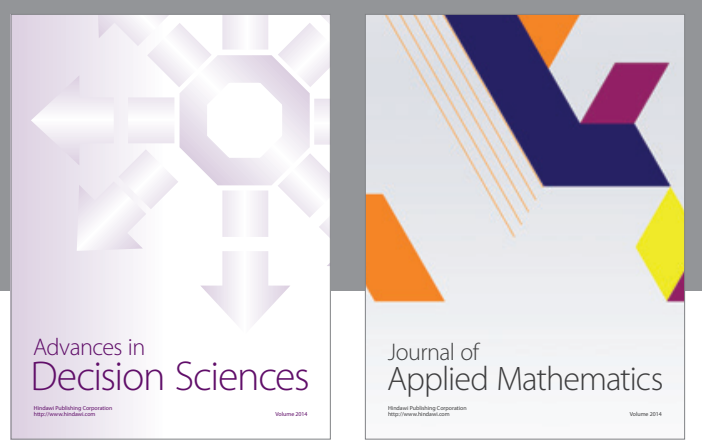

Algebra

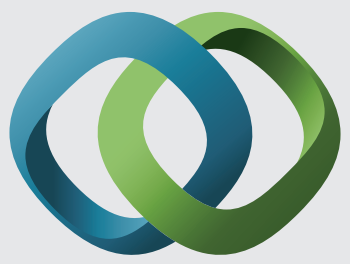

\section{Hindawi}

Submit your manuscripts at

http://www.hindawi.com
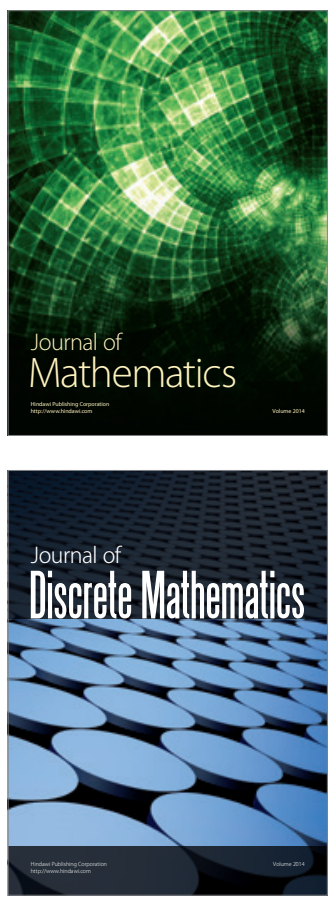

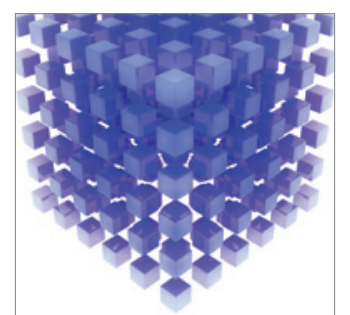

Mathematical Problems in Engineering
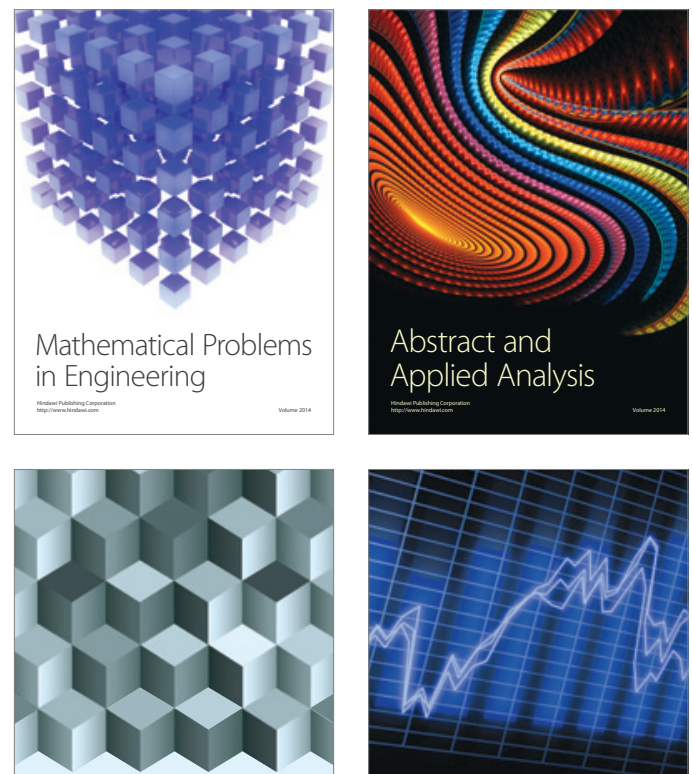

Journal of

Function Spaces

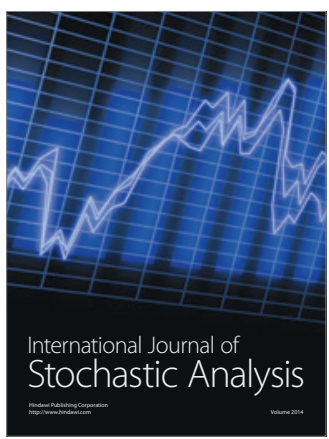

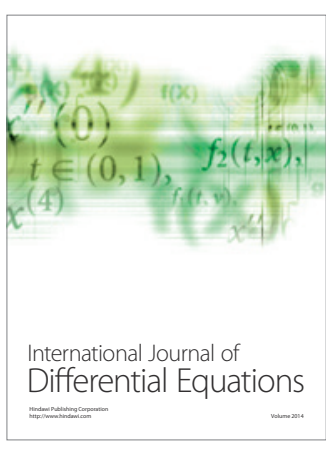
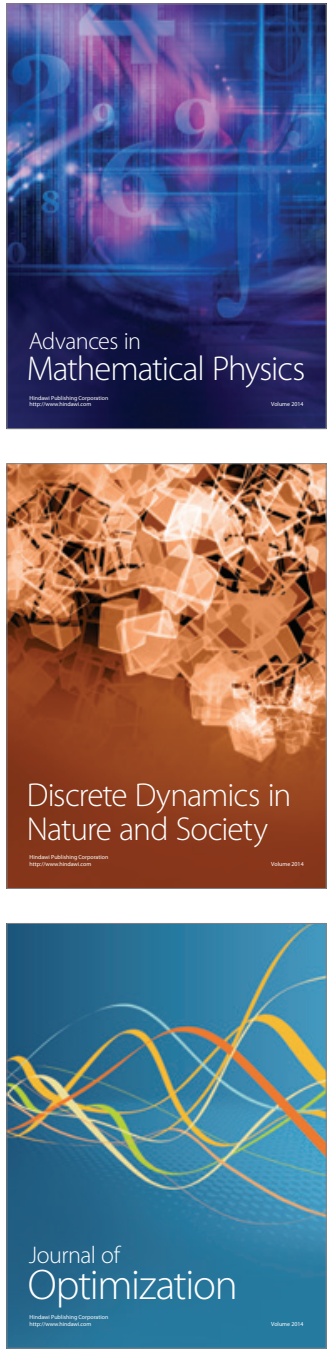